BMJ Paediatrics Open

\title{
New anthropometric classification scheme of preoperative nutritional status in children: a retrospective observational cohort study
}

Anne Stey, ${ }^{1}$ Joni Ricks-Oddie, ${ }^{2}$ Sheila Innis, ${ }^{3}$ Shawn J Rangel, ${ }^{4}$ R Lawrence Moss, ${ }^{5}$ Bruce L Hall, ${ }^{6,7,8}$ Albert Dibbins, ${ }^{9}$ Erik D Skarsgard ${ }^{3}$

\section{ABSTRACT}

Objective WHO uses anthropometric classification scheme of childhood acute and chronic malnutrition based on low body mass index (BMI) ('wasting') and height for age ('stunting'), respectively. The goal of this study was to describe a novel two-axis nutritional classification scheme to (1) characterise nutritional profiles in children undergoing abdominal surgery and (2) characterise relationships between preoperative nutritional status and postoperative morbidity.

Design This was a retrospective observational cohort study. Setting The setting was 50 hospitals caring for children in North America that participated in the American College of Surgeons National Surgical Quality Improvement Program Paediatric from 2011 to 2013.

Participants Children $>28$ days who underwent major abdominal operations were identified.

Interventions/main predictor The cohort of children was divided into five nutritional profile groups based on both BMI and height for age Z-scores: (1) underweight/ short, (2) underweight/tall, (3) overweight/short, (4) overweight/tall and (5) non-outliers (controls).

Main outcome measures Multiple variable logistic regressions were used to quantify the association between 30-day morbidity and nutritional profile groups while adjusting for procedure case mix, age and American Society of Anaesthesiologists class.

Results A total of 39520 cases distributed as follows: underweight/short (656, 2.2\%); underweight/tall (252, 0.8\%); overweight/short $(733,2.4 \%)$ and overweight/tall (1534, $5.1 \%)$. Regression analyses revealed increased adjusted odds of composite morbidity (35\%) and reintervention events $(75 \%)$ in the underweight/short group, while overweight/ short patients had increased adjusted odds of composite morbidity and healthcare-associated infections (43\%), and reintervention events $(79 \%)$ compared with controls.

Conclusion Stratification of preoperative nutritional status using a scheme incorporating both $\mathrm{BMl}$ and height for age is feasible. Further research is needed to validate this nutritional risk classification scheme for other surgical procedures in children.

\section{INTRODUCTION}

Malnutrition (both overnutrition and undernutrition) is prevalent among hospitalised children. ${ }^{1}$ A variety of paediatric nutritional
What is already known on this topic?

Nutritional status is an important predictor of health in growing children. This has been difficult to quantify objectively in children due to their variable.

\section{What this study hopes to add?}

This study proposes a stratification of preoperative nutritional status using a scheme incorporating both body mass index and height for age and using this system suggests that underweight short and overweight short children appear to be at greatest risk of postoperative morbidity.

screening tools which combine subjective assessment with objective anthropometric measurements reflecting body composition exist. ${ }^{2-4}$ There is a clear need to develop valid measures of general nutritional status in children as potentially modifiable risk factors for healthcare outcomes.

Existing anthropometric classification schemes define the nutritional status of children relative to standardised populations into three pathological states: wasting (low body mass index (BMI)), stunting (low height for age) and overweight/obese (high BMI). ${ }^{5}{ }^{6}$ Hospitalised children, especially those who require surgery, may require a more detailed classification system that accounts for both their general nutritional state and disease-specific impact on growth, as a predictor of adverse postoperative outcomes.

A recent evidence review of nutritional assessment measures and clinical outcomes in children undergoing surgery confirms a gap in valid outcome predictors. ${ }^{7}$ One major challenge to anthropometric measurements as reliable predictors of nutritional state in children are the confounding effects of disease, 
its treatment and related comorbidities (including congenital anomalies) on weight and stature.

The aim of this study was to create and describe a novel, anthropometric measure of preoperative nutritional status using a two-axis classification scheme that incorporates both gender-specific BMI and height for age. The primary objective was to describe the distribution of a large cohort of children undergoing abdominal surgery using this novel two-axis classification scheme. The secondary objective was to determine if specific nutritional profile groups had higher odds of postoperative morbidity. We hypothesised that a more a detailed two-axis classification scheme would identify 'at risk' nutritional profiles that might be overlooked by the classic BMI or height for age anthropometric classification alone.

\section{METHODS}

\section{Data source and patient sample}

This retrospective observational cohort study used 2011-2013 American College of Surgeons National Surgical Quality Improvement Program-Paediatric (ACS NSQIP-P) data from 54 participating ACS NSQIP-P hospitals across North America. The dataset includes strictly defined preoperative patient demographic, clinical and procedural variables, as well as postoperative adverse events. Trained, surgical clinical reviewers collected ACS NSQIP-P data from the medical record, and via follow-up patient/family phone calls in the absence of documented encounters, to ascertain 30-day postoperative morbidity with excellent capture. ${ }^{78}$

The inclusion criteria were children from 29 days to 18 years of age undergoing major abdominal procedures at the ACS-NSQIP-P centres in 2011-2103 as specified by the current procedural terminology (CPT, AMA) codes (online supplementary file 1). All major abdominal procedures accrued at participating institutions over the 3-year time period were included.

\section{Measures}

The main predictor variable was a new categorical fivelevel anthropometric measure of preoperative nutritional status using a two-axis classification scheme that incorporates both gender-specific BMI and height for age. The patient preoperative weight, height, age and gender data in the 2011-13 ACS NSQIP-P dataset were used to calculate gender-specific BMI and height Z-scores. Z-scores were calculated based on the $\mathrm{WHO}^{9}$ algorithm for children under 2 years of age BMI Z-score, which in these children is based on recumbent length rather than stature. The Centre for Disease Control provides an algorithm ${ }^{10}$ for children 2 years of age and older which uses BMI. The BMI and height Z-scores were used to assign children to one of four 'dual outlier' groups (Z-scores $<-2$ or $>2$ for both BMI and height axes) as follows: (1) underweight/ short, (2) underweight/tall, (3) overweight/short, (4) overweight/tall and (5) controls, as shown in figure 1.
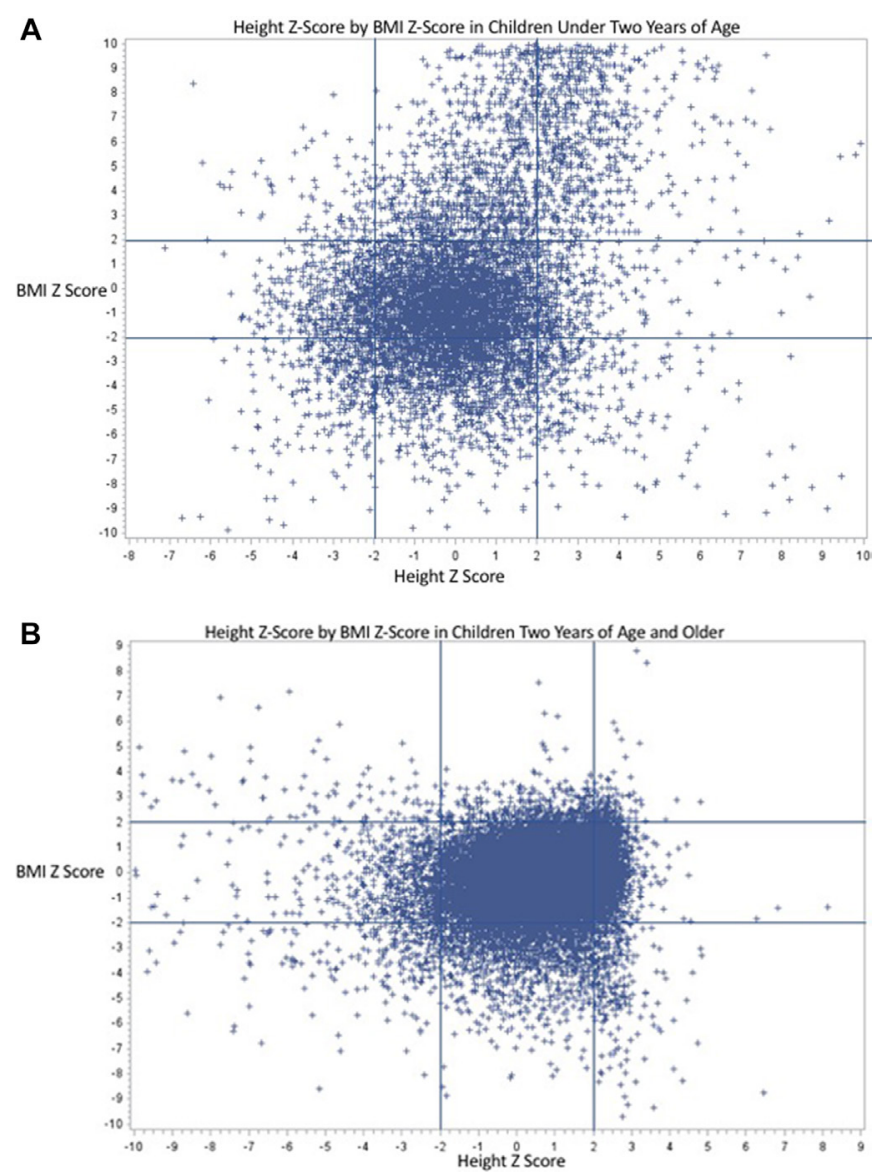

Figure 1 (A, B) Children from 29 days to 18 years were divided into five nutritional profile groups based on the WHO and the Centre for Disease Control (CDC) growth curve algorithms and plotted by their assigned body mass index (BMI) Z-score along the $\mathrm{Y}$ axis and height Z-score along the $X$ axis. Children outside of two Z-scores in any direction were categorised as an outlier nutritional profile group. (A) Children under 2 years of age using the $\mathrm{WHO}$ algorithm to assign Z-scores. (B) Children 2 years of age and older using the CDC algorithm to assign Z-scores.

\section{Statistical analysis}

Descriptive counts and frequencies of preoperative clinical and procedural variables as well as postoperative 30-day morbidity for the five nutritional profile groups were calculated. All patient-specific independent variables were dichotomous with the exception of American Society of Anaesthesiologists (ASA) class, age, race and preoperative sepsis. ASA class was categorised using ASA class I as reference and was treated as a continuous numeric variable in multivariate analysis. Age was categorised as infants $<1$ year, $1-2$ years, $3-5$ years, $6-7$ years, 8-12 years and 13-18 years. In the multivariate analysis, age was treated as a continuous variable. Race was categorised as Caucasian, African-American, Asian, Native American and other (which included patients where race was missing) with Caucasian as reference. Preoperative sepsis was categorised as no sepsis, systemic inflammatory response syndrome, sepsis and septic shock. 
The main outcomes were complications occurring within 30 days of surgery including surgical-site infection, postoperative sepsis, return to the operating room, wound dehiscence, transfusion within 72 hours, renal failure, pneumonia, cardiac arrest, deep vein thrombosis, urinary tract infection and mortality. Three binary composite dependent variables were created because the number of patients having any single complication was low. These included (1) composite morbidity, defined as one or more of the following: surgical-site infection, pneumonia, reintubation, pulmonary embolism, renal insufficiency, urinary tract infection, coma, seizure, peripheral nerve injury, intraventricular haemorrhage, intracranial haemorrhage, cardiac arrest, intraoperative and postoperative transfusion within 72 hours, graft failure, venous thrombosis requiring therapy, postoperative sepsis and central line-associated blood stream infection; (2) healthcare-associated infection (HAI), defined as one or more of the following: surgical-site infection, postoperative sepsis, pneumonia, central line-associated infection and urinary tract infection; and (3) need for re-intervention, defined as one or more of the following: unplanned reoperation, unplanned reintubation, acute renal failure requiring dialysis and cardiac arrest. Mortality was not specifically analysed due to the rarity of this occurrence.

Height was missing in 9483 out of 39520 children, approximately $24 \%$ of the study sample, and would have been a major source of bias. The missingness of height was assumed to be random, based on the comparability of preoperative patient variables and postoperative 30-day morbidity between children with or without height records (data not shown). Multiple imputation (a total of 20) using multivariate normal with data augmentation was done to directly impute nutrition profile groups rather than height alone. Weight was missing in $<5 \%$ of the sample and was dealt with in a similar manner.

Pearson R was calculated for all independent preoperative patient variables and anthropometric Z-scores. Independent preoperative patient variables that were correlated more than 0.7 correlation (either direction) with either preoperative BMI Z-score or preoperative height Z-score were included as auxiliary variables in the multiple imputation model. The final regression model included 37 independent preoperative patient and procedure variables listed in online supplementary file 2 and adjusted for clustering at the hospital level using random effects. Three models were run for each of the three outcomes. Subsequently, separate logistic regression models with random effects were performed to specifically assess nutrition profile groups as a predictor of composite 30-day morbidity, HAI and reintervention events, while adjusting for procedure case mix (CPT linear risk), ${ }^{11}$ age (as a numeric variable derived from age) and ASA class treated as continuous variables to facilitate model convergence. These variables were selected based on previous studies which demonstrated that they account for the majority of variance in ACS NSQIP-P 30-day morbidity. ${ }^{12} 13$
Two sensitivity analyses were performed. First, all ex-premature children were excluded and the regression analyses repeated, to interrogate potential bias caused by prematurity on growth potential. The second sensitivity analysis excluded all emergency and urgent cases to assess potential bias in favour of higher complication rates associated with emergency cases. All data management and analyses were performed in SAS V.9.3 (SAS Institute).

\section{RESULTS}

A total of 39520 children were analysed. Of the 30037 children with complete anthropometric data, 3175 children $(10.5 \%)$ could be categorised into one of four nutritional profile groups defined by dual outlier status, for both BMI and height for age. The largest outlier category was overweight tall, the smallest outlier category was underweight tall. The scatter plot is depicted in figure 1 and demonstrates a skewness within the population, with disproportionately more children having negative height Z-scores.

Compared with the other groups, underweight short children had associated risk factors suggesting nutritional vulnerability (highest rates of preoperative nutritional supplementation and weight loss $>10 \%$ bodyweight in the 6 months before surgery), and higher rates of comorbidity (table 1). These included higher rates of premature birth, oesophageal-gastrointestinal disease, neurological comorbidity, developmental delay and a history of cardiac surgery. Underweight short children also had higher unadjusted rates of specific adverse outcomes including postoperative sepsis, need for postoperative transfusion and mortality, as well as the highest rates of composite morbidity and need for reintervention (table 2).

When controlling for procedure case mix, age and ASA, underweight short and overweight short children had $35 \%$ and $43 \%$ increased adjusted odds of 30-day composite morbidity compared with children who were within two Z-scores of mean BMI and height, respectively (table 3), a finding which persisted when prematurely born children were excluded. However, when urgent/ emergent cases were excluded underweight short children no longer had higher adjusted odds of 30-day composite morbidity. Multivariate analysis also revealed that overweight short children had a $43 \%$ increased adjusted odds of developing a HAI compared with children who were within two Z-scores of mean BMI and height (table 4). Both underweight short and overweight short children also demonstrated significantly increased adjusted odds of need for reintervention compared with children who were within two Z-scores of mean BMI and height ( $75 \%$ and $79 \%$, respectively; table 5 ).

Adding the four nutritional profile groups to procedure case mix, age and ASA for modelling composite morbidity increased the area under the receiver operating characteristic curve from 0.70 to 0.71 . Similarly, the four nutritional profile groups added to the procedure 
Open access

Table 1 Preoperative patient specific clinical variables and comorbidities by nutritional profile group

\begin{tabular}{|c|c|c|c|c|c|c|}
\hline Preoperative clinical variables & $\begin{array}{l}\text { Underweight } \\
\text { short } n=656\end{array}$ & $\begin{array}{l}\text { Overweight } \\
\text { short } n=733\end{array}$ & $\begin{array}{l}\text { Underweight } \\
\text { tall } n=252\end{array}$ & $\begin{array}{l}\text { Overweight } \\
\text { tall } n=1534\end{array}$ & $\begin{array}{l}\text { Normal } \\
n=26862\end{array}$ & P values* \\
\hline Inpatient status & 585 (89.2) & $591(80.6)$ & 210 (83.3) & $1142(74.5)$ & $20280(75.5)$ & $<0.0001$ \\
\hline Case status & & & & & & $<0.0001$ \\
\hline Elective & $498(76.0)$ & $411(56.1)$ & $98(38.9)$ & $1240(80.8)$ & $14431(53.7)$ & \\
\hline Urgent & 87 (13.3) & $151(20.6)$ & $54(21.4)$ & $124(8.1)$ & $4851(18.1)$ & \\
\hline Emergent & $71(10.9)$ & $171(23.3)$ & $100(39.7)$ & $170(11.1)$ & $7580(28.2)$ & \\
\hline Male gender & $405(61.7)$ & $453(61.8)$ & $156(61.9)$ & 969 (63.2) & $14911(55.5)$ & $<0.0001$ \\
\hline Race & & & & & & 0.0003 \\
\hline Caucasian & $491(79.2)$ & 567 (81.9) & $185(76.1)$ & $1240(81.5)$ & $21283(83.8)$ & \\
\hline African-American & $110(17.7)$ & $105(15.2)$ & $49(20.2)$ & $239(15.7)$ & $3289(13.0)$ & \\
\hline Asian & $18(2.9)$ & $18(2.6)$ & $9(3.7)$ & $33(2.2)$ & $661(2.6)$ & \\
\hline Native American & $1(0.2)$ & $2(0.3)$ & 0 & $8(0.5)$ & $120(0.5)$ & \\
\hline Hispanic & $63(9.6)$ & $132(18.0)$ & $40(15.9)$ & $199(13.0)$ & $4054(15.1)$ & 0.04 \\
\hline Age & & & & & & $<0.0001$ \\
\hline 29-364 days & $308(47.0)$ & $225(30.7)$ & $95(37.7)$ & $840(54.8)$ & 5069 (18.9) & \\
\hline $1-2$ years & $32(4.9)$ & $127(17.3)$ & $13(5.2)$ & $508(33.1)$ & $1431(5.3)$ & \\
\hline 3-5years & $53(8.1)$ & $122(16.6)$ & $61(24.2)$ & $24(1.6)$ & 3007 (11.2) & \\
\hline $6-7$ years & 32 (4.9) & $45(6.1)$ & 36 (14.3) & $31(2.0)$ & 2117 (7.9) & \\
\hline $8-12$ years & $97(14.9)$ & $114(15.6)$ & $38(15.1)$ & $83(5.4)$ & $6980(26.0)$ & \\
\hline 13-18years & $134(20.4)$ & $100(13.6)$ & $9(3.6)$ & $48(3.1)$ & $8258(30.7)$ & \\
\hline Premature birth & 170 (25.9) & $94(12.8)$ & $14(5.6)$ & $261(17.0)$ & 2276 (8.5) & $<0.0001$ \\
\hline \multicolumn{7}{|l|}{ Metabolic and nutritional conditions } \\
\hline Nutritional support & $253(38.6)$ & $139(19.0)$ & $29(11.5)$ & $286(18.6)$ & $2515(9.4)$ & $<0.0001$ \\
\hline $\begin{array}{l}\text { Greater than } 10 \% \text { weight loss } \\
\text { within } 6 \text { months }\end{array}$ & $286(43.6)$ & $72(9.8)$ & $37(14.7)$ & $155(10.1)$ & $1823(6.8)$ & $<0.0001$ \\
\hline
\end{tabular}

Renal conditions

\begin{tabular}{|c|c|c|c|c|c|c|}
\hline Acute renal failure & $5(0.8)$ & $3(0.4)$ & $2(0.8)$ & $11(0.7)$ & $126(0.5)$ & 0.27 \\
\hline \multicolumn{7}{|c|}{ American Society of Anesthesiologists class } \\
\hline I & $33(5.0)$ & $181(24.7)$ & $74(29.4)$ & $275(17.9)$ & $7904(29.4)$ & $<0.0001$ \\
\hline II & $147(22.4)$ & $261(35.6)$ & $111(44.1)$ & $673(43.9)$ & $12044(44.8)$ & \\
\hline III & $407(62.0)$ & $251(34.2)$ & $59(23.4)$ & $514(33.5)$ & $6294(23.4)$ & \\
\hline IV & $65(9.9)$ & $39(5.3)$ & $6(2.4)$ & $66(4.3)$ & $591(2.2)$ & \\
\hline V & $4(0.6)$ & $1(0.1)$ & $2(0.8)$ & $6(0.4)$ & $29(0.1)$ & \\
\hline \multicolumn{7}{|l|}{ Gastrointestinal conditions } \\
\hline $\begin{array}{l}\text { Oesophageal gastrointestinal } \\
\text { disease }\end{array}$ & $436(66.5)$ & $331(45.2)$ & $100(39.7)$ & $743(48.4)$ & $8821(32.8)$ & $<0.0001$ \\
\hline Hepatobiliary disease & $35(5.3)$ & $36(4.9)$ & $5(2.0)$ & $76(5.0)$ & $2321(8.6)$ & $<0.0001$ \\
\hline \multicolumn{7}{|l|}{ Cardiovascular conditions } \\
\hline Cardiac risk factors & & & & & & $<0.0001$ \\
\hline Mild cardiac risk factors & $103(15.7)$ & $73(10.0)$ & $5(2.0)$ & $173(11.3)$ & $1444(5.4)$ & \\
\hline Moderate cardiac risk factors & $55(8.4)$ & $42(5.7)$ & $6(2.4)$ & $72(4.7)$ & $693(2.6)$ & \\
\hline Severe cardiac risk factors & $26(4.0)$ & $14(1.9)$ & $1(0.4)$ & $45(2.9)$ & $280(1.0)$ & \\
\hline Cardiac surgery & $81(12.4)$ & $56(7.6)$ & $9(3.6)$ & $127(8.3)$ & $1086(4.0)$ & $<0.0001$ \\
\hline \multicolumn{7}{|l|}{ Neurological conditions } \\
\hline Cerebrovascular accident & $42(6.4)$ & $30(4.1)$ & $5(2.0)$ & $63(4.1)$ & 509 (1.9) & $<0.0001$ \\
\hline Seizure disorder & $124(18.9)$ & $65(8.9)$ & $20(7.9)$ & $72(4.7)$ & $1110(4.1)$ & $<0.0001$ \\
\hline Cerebral palsy & $112(17.1)$ & $22(3.0)$ & $8(3.2)$ & $16(1.0)$ & $516(1.9)$ & $<0.0001$ \\
\hline
\end{tabular}


Table 1 Continued

\begin{tabular}{|c|c|c|c|c|c|c|}
\hline Preoperative clinical variables & $\begin{array}{l}\text { Underweight } \\
\text { short } n=656\end{array}$ & $\begin{array}{l}\text { Overweight } \\
\text { short } n=733\end{array}$ & $\begin{array}{l}\text { Underweight } \\
\text { tall } n=252\end{array}$ & $\begin{array}{l}\text { Overweight } \\
\text { tall } n=1534\end{array}$ & $\begin{array}{l}\text { Normal } \\
\mathrm{n}=26862\end{array}$ & $\mathbf{P}_{\text {values* }}^{*}$ \\
\hline Neuromuscular disorder & $77(11.7)$ & $45(6.1)$ & $12(4.8)$ & $59(3.9)$ & $844(3.1)$ & $<0.0001$ \\
\hline Developmental delay & $263(40.1)$ & $147(20.1)$ & $26(10.3)$ & $249(16.2)$ & $2786(10.4)$ & $<0.0001$ \\
\hline $\begin{array}{l}\text { Central nervous system structural } \\
\text { abnormality }\end{array}$ & $127(19.4)$ & $75(10.2)$ & $14(5.6)$ & $156(10.2)$ & $1296(4.8)$ & $<0.0001$ \\
\hline \multicolumn{7}{|c|}{ Haematological and immunological conditions } \\
\hline Bleeding disorder & $11(1.7)$ & $9(1.2)$ & $0(0)$ & $16(1.0)$ & $223(0.8)$ & 0.025 \\
\hline Steroid use within 30 days & $37(5.6)$ & $33(4.5)$ & $4(1.6)$ & $50(3.3)$ & $918(3.4)$ & 0.007 \\
\hline Immune disease & $18(2.7)$ & $18(2.5)$ & $0(0)$ & $19(1.2)$ & $615(2.3)$ & 0.69 \\
\hline Haematological disorder & $64(9.8)$ & $37(5.1)$ & $8(3.2)$ & $73(4.8)$ & $1396(5.2)$ & 0.001 \\
\hline Bone marrow transplant & $4(0.6)$ & $2(0.3)$ & 0 & $6(0.4)$ & $79(0.3)$ & 0.33 \\
\hline Transplantation & $4(0.6)$ & $4(0.6)$ & 0 & $3(0.2)$ & $147(0.6)$ & 0.61 \\
\hline $\begin{array}{l}\text { Preoperative red blood cell } \\
\text { transfusion }\end{array}$ & $25(3.8)$ & $19(2.6)$ & $5(2.0)$ & $23(1.5)$ & $384(1.4)$ & $<0.0001$ \\
\hline \multicolumn{7}{|l|}{ Pulmonary conditions } \\
\hline Asthma & $43(6.6)$ & $70(9.6)$ & $7(2.8)$ & $81(5.3)$ & $1665(6.2)$ & 0.12 \\
\hline Chronic lung disease & $92(14.0)$ & $56(7.6)$ & $5(2.0)$ & $112(7.3)$ & $856(3.2)$ & $<0.0001$ \\
\hline Cystic fibrosis & $8(1.2)$ & $4(0.6)$ & $1(0.4)$ & $7(0.5)$ & $181(0.7)$ & 0.52 \\
\hline Structural pulmonary abnormality & $83(12.7)$ & $64(8.7)$ & $11(4.4)$ & $130(8.5)$ & $1078(4.0)$ & $<0.0001$ \\
\hline Oxygen supplementation & $70(10.7)$ & $53(7.2)$ & $10(4.0)$ & $96(6.3)$ & $691(2.6)$ & $<0.0001$ \\
\hline Tracheostomy & $18(2.7)$ & $30(4.1)$ & $3(1.2)$ & $37(2.4)$ & $264(1.0)$ & $<0.0001$ \\
\hline \multicolumn{7}{|l|}{ Acuity of condition } \\
\hline Prior surgery within 30 days & $31(4.7)$ & $22(3.0)$ & $10(4.0)$ & $39(2.5)$ & $530(2.0)$ & $<0.0001$ \\
\hline Open wound & $22(3.4)$ & $13(1.8)$ & $4(1.6)$ & $40(2.6)$ & $538(2.0)$ & 0.07 \\
\hline Cardiopulmonary resuscitation & $2(0.3)$ & $3(0.4)$ & $1(0.4)$ & $2(0.1)$ & $28(0.1)$ & 0.006 \\
\hline Do not resuscitate & $2(0.3)$ & 0 & 0 & $1(0.1)$ & $21(0.1)$ & 0.35 \\
\hline Inotropic support & $15(2.3)$ & $7(1.0)$ & $2(0.8)$ & $11(0.7)$ & $179(0.7)$ & $<0.0001$ \\
\hline Preoperative sepsis within 48 hours & & & & & & $<0.0001$ \\
\hline No sepsis & $620(94.5)$ & $645(88.0)$ & $205(81.4)$ & $1452(94.7)$ & $23022(85.7)$ & \\
\hline $\begin{array}{l}\text { Systemic inflammatory } \\
\text { response syndrome }\end{array}$ & $17(2.6)$ & $38(5.2)$ & $16(6.4)$ & $36(2.4)$ & $1980(7.4)$ & \\
\hline Sepsis & $13(2.0)$ & $47(6.4)$ & $31(12.3)$ & $39(2.5)$ & $1799(6.7)$ & \\
\hline Septic shock & $6(0.9)$ & $3(0.4)$ & $0(0)$ & $7(0.5)$ & $61(0.2)$ & \\
\hline
\end{tabular}

${ }^{\star}$ Derived from $\chi^{2}$ test of preoperative patient-specific clinical variables among nutritional profile groups.

case mix, age and ASA for modelling HAI increased the area under the receiver operating characteristic curve from 0.67 to 0.68 . The area under the receiver operating curve did not change with the addition of four nutritional profile groups for reinterventions, 0.73 to 0.73 .

\section{DISCUSSION}

In contrast to adults, the evidence of a predictive relationship between nutritional state and healthcare outcomes in children is sparse. The severity of malnutrition assessed with a variety of tools including estimates of energy intake and body composition, serum markers and anthropometric measurements have been shown to have some correlation with outcomes in critically ill children, ${ }^{14} 15$ and paediatric cardiac surgery patients. ${ }^{16-19}$ Yet no nutrition metric that is generally predictive of outcome for populations of hospitalised children has been identified.

Only a few studies in children undergoing non-cardiac surgery have sought an association between anthropometric classification and postoperative morbidity. Previous studies using the aggregate NSQIP paediatric dataset have shown that children in the $\leq 5$ th weight percentile experienced higher rates of postoperative transfusion and reintubation, ${ }^{20}$ while children undergoing appendectomy ${ }^{21}$ and urological procedures ${ }^{22}$ who met BMI percentile definitions of overweight/obese were more likely to experience postoperative wound complications. However, a limitation of existing anthropometric 
Table 2 Unadjusted rates of postoperative 30-day complication by nutritional profile group

\begin{tabular}{|c|c|c|c|c|c|c|}
\hline Postoperative 30 -day complications & $\begin{array}{l}\text { Underweight } \\
\text { short } n=656\end{array}$ & $\begin{array}{l}\text { Overweight } \\
\text { short } n=733\end{array}$ & $\begin{array}{l}\text { Underweight } \\
\text { tall } n=252\end{array}$ & $\begin{array}{l}\text { Overweight } \\
\text { tall } n=1534\end{array}$ & $\begin{array}{l}\text { Normal } \\
n=26862\end{array}$ & P values* \\
\hline Surgical-site infection & $13(2.0)$ & $34(4.6)$ & $6(2.4)$ & $41(2.7)$ & $913(3.4)$ & 0.31 \\
\hline Postoperative sepsis & $16(2.4)$ & $6(0.8)$ & $1(0.4)$ & $26(1.7)$ & $224(0.8)$ & 0.0002 \\
\hline Return to operating room & $32(4.9)$ & $39(5.3)$ & $5(2.0)$ & $64(4.2)$ & 769 (2.9) & $<0.0001$ \\
\hline Dehiscence & $4(0.6)$ & $6(0.8)$ & $3(1.2)$ & $10(0.7)$ & $111(0.4)$ & 0.03 \\
\hline Transfusion within 72 hours & $16(2.4)$ & $7(1.0)$ & $3(1.2)$ & $19(1.2)$ & $162(0.6)$ & $<0.0001$ \\
\hline Reintubation & $16(2.4)$ & $5(0.7)$ & $3(1.2)$ & $7(0.5)$ & $106(0.4)$ & $<0.0001$ \\
\hline Acute renal failure & $2(0.3)$ & $2(0.3)$ & $0(0)$ & $3(0.2)$ & $34(0.1)$ & 0.18 \\
\hline Central line-associated infection & $3(0.5)$ & $1(0.1)$ & 0 & $6(0.4)$ & $51(0.2)$ & 0.24 \\
\hline Pneumonia & $8(1.2)$ & $7(1.0)$ & $2(0.8)$ & $7(0.5)$ & $135(0.5)$ & 0.01 \\
\hline Cardiac arrest & $3(0.5)$ & $1(0.1)$ & $1(0.4)$ & $5(0.3)$ & $35(0.1)$ & 0.02 \\
\hline Deep vein thrombosis & $1(0.2)$ & $1(0.1)$ & $1(0.4)$ & $4(0.3)$ & $56(0.2)$ & 0.82 \\
\hline Urinary tract infections & $4(0.6)$ & $5(0.7)$ & $0(0)$ & $11(0.7)$ & $155(0.6)$ & 0.84 \\
\hline Mortality & $4(0.6)$ & $3(0.4)$ & $1(0.4)$ & $5(0.3)$ & $51(0.2)$ & 0.005 \\
\hline Composite 30-day morbidity & $66(10.1)$ & $61(8.3)$ & $20(7.9)$ & $107(7.0)$ & $1634(6.1)$ & $<0.0001$ \\
\hline Healthcare-associated infections & $34(5.2)$ & $46(6.3)$ & $9(3.6)$ & 79 (5.2) & $1287(4.8)$ & 0.18 \\
\hline Reintervention events & $48(7.3)$ & $43(5.9)$ & $8(3.2)$ & $71(4.6)$ & $881(3.3)$ & $<0.0001$ \\
\hline
\end{tabular}

${ }^{*}$ Derived from a $\chi^{2}$ test comparing complications among nutritional profile group.

classification schemes is that they were developed to define the nutritional state of an individual relative to a reference population of 'healthy' children. Hospitalised children, and notably those undergoing abdominal surgery represent a heterogeneous population, some of whom are healthy with simple surgical conditions like appendicitis, while others have a diverse variety of acute or chronic diseases, often with significant comorbidities who may not conform to the classic malnutrition categories of 'wasting' (low BMI), 'stunting' (low height for age) and "overweight/obese (high BMI). Therefore, classification schemes which more accurately capture

Table 3 Multivariate logistic regression predicting composite 30-day morbidity

\begin{tabular}{|c|c|c|c|}
\hline & OR $^{*}$ & $95 \% \mathrm{Cl}^{*}$ & P values* \\
\hline $\begin{array}{l}\text { Composite morbidity } \\
\text { current procedural } \\
\text { terminology linear risk }\end{array}$ & 0.38 & 0.36 to 0.40 & $<0.0001$ \\
\hline Age & 0.99 & 0.98 to 1.00 & 0.07 \\
\hline $\begin{array}{l}\text { American Society of } \\
\text { Anaesthesiologists } \\
\text { class }\end{array}$ & 1.02 & 1.00 to 1.04 & 0.06 \\
\hline Underweight short & 1.35 & 1.03 to 1.75 & 0.04 \\
\hline Overweight short & 1.43 & 1.06 to 1.89 & 0.01 \\
\hline Underweight tall & 1.32 & 0.81 to 2.22 & 0.30 \\
\hline Overweight tall & 1.00 & 0.81 to 1.25 & 0.97 \\
\hline
\end{tabular}

Both composite 30-day morbidity current procedural terminology linear risk and age were continuous variables.

*Derived from Proc Mianalyze following multiple imputation of the four nutritional profile groups using Proc Glimmix. the effects of nutritional state and underlying disease on growth patterns are required.

The current study demonstrates the feasibility of an anthropometric classification scheme that combines BMI on the $\mathrm{Y}$ axis and height for age on the $\mathrm{X}$ axis as a means of substratifying outliers into four nutritional profile groups . Underweight short and overweight short patients had higher postoperative composite morbidity and need for reintervention after controlling for case mix, age and ASA. Underweight short children also had higher rates

Table 4 Multivariate logistic regression predicting composite healthcare-associated infections

\begin{tabular}{llll} 
& OR $^{*}$ & $\mathbf{9 5 \%} \mathbf{C l}^{*}$ & $\mathbf{P}^{*}$ values \\
\hline $\begin{array}{l}\text { Composite healthcare- } \\
\text { associated infection } \\
\text { current procedural } \\
\text { terminology linear risk }\end{array}$ & 0.33 & 0.30 to 0.36 & $<0.0001$ \\
\hline $\begin{array}{l}\text { Age } \\
\text { American Society of }\end{array}$ & 0.98 & 0.97 to 0.99 & 0.0001 \\
$\begin{array}{l}\text { Anaesthesiologists } \\
\text { class }\end{array}$ & 1.05 & 1.02 to 1.08 & $<0.0001$ \\
$\begin{array}{l}\text { Underweight short } \\
\text { Overweight short }\end{array}$ & 0.89 & 0.60 to 1.33 & 0.56 \\
\hline $\begin{array}{l}\text { Underweight tall } \\
\text { Overweight tall }\end{array}$ & 0.75 & 0.37 to 1.52 & 0.42 \\
\hline
\end{tabular}

Both composite healthcare-associated infection current procedural terminology linear risk and age were continuous variables.

*Derived from Proc Mianalyze following multiple imputation of the four nutritional profile groups using Proc Glimmix. 
Table 5 Multivariate logistic regression predicting composite reintervention events

\begin{tabular}{|c|c|c|c|}
\hline & OR $^{\star}$ & $95 \% \mathrm{Cl}^{*}$ & P values* \\
\hline $\begin{array}{l}\text { Composite } \\
\text { reintervention events } \\
\text { current procedural } \\
\text { terminology linear risk }\end{array}$ & 0.34 & 0.31 to 0.36 & $<0.0001$ \\
\hline Age & 0.99 & 0.98 to 1.00 & 0.28 \\
\hline $\begin{array}{l}\text { American Society of } \\
\text { Anaesthesiologists } \\
\text { class }\end{array}$ & 1.01 & 0.98 to 1.01 & 0.35 \\
\hline Underweight short & 1.75 & 1.28 to 2.38 & 0.001 \\
\hline Overweight short & 1.79 & 1.30 to 2.50 & 0.001 \\
\hline Underweight tall & 0.99 & 0.47 to 2.08 & 0.97 \\
\hline Overweight tall & 1.08 & 0.82 to 1.41 & 0.58 \\
\hline
\end{tabular}

Both composite reintervention events current procedural terminology linear risk and age were continuous variables. ${ }^{*}$ Derived from Proc Mianalyze following multiple imputation of the four nutritional profile groups using Proc Glimmix.

of associated disease that could have contributed to their preoperative nutritional state, and likely also had some influence on the occurrence of adverse outcomes. The other distinguishing characteristic of the underweight short group were significantly undernourished prior to surgery.

Overweight short patients, on the other hand, were at increased risk for all three adverse outcomes: composite morbidity, hospital-acquired infection (most commonly surgical-site infection) and need for reintervention. In contrast to the underweight short group, obese short patients did not have distinguishing comorbidity profiles. Potential body morphology determinants include endocrine/metabolic disorders, and genetic disorders/ congenital anomalies which could disturb musculoskeletal growth leading to reduced stature. Finally, severe acute or chronic inflammatory diseases of the gastrointestinal tract may have confounding effects on both the patient's nutritional state and body morphology (eg, effects of chronic steroid exposure, total parenteral nutrition, oedema from low protein states). Some combination of the patient's underlying nutrition, the metabolic/ inflammatory activity of the disease requiring surgery and the failure of medical treatments prior to a decision for surgery contribute to adverse postopertiave outcomes. Body morphology as captured by the four nutritional profile groups described may be a proxy medical and nutritional status .

There are several limitations to this study. This was a retrospective secondary data analysis of observational data, and is therefore subject to inherent bias, particularly with regards to missing data. A second limitation is the lack of specificity of the composite morbidity outcomes. Despite the relatively large number of children analysed, the very low rates of postoperative morbidity in children means that the numbers of any individual outcome are small, which increases the probability of type 2 errors. The three composite morbidities intended to capture any 30-day composite morbidity, HAIs and reintervention events are an attempt to create some granularity in the type of morbidity associated with certain nutritional profile groups while allowing modest aggregation. ${ }^{23}$ Another limitation is that the ACS NSQIP-P dataset categorises patients by surgical procedure rather than diagnosis, which limits the discernment of growth disturbances by disease states. A fourth limitation is the assumption that the height data are missing at random and the use of multiple imputation. This could have quite a large effect on our outcomes and may limit our ability to predict different outcomes based on a score where a portion of the primary predictor was imputed. Finally, as demonstrated by the high rate of underweight and overweight children, this study focused on a highly select group of children referred to tertiary children's hospitals which have self-selected to participate in ACS NSQIP-P. As such the findings of this study may not be generalisable to a broader cohort of hospitals or hospitalised children.

This study demonstrates that it is feasible to stratify children into four anthropometric risk groups based on height and weight. Two of these groups, underweight short and overweight short, had significantly higher adjusted 30-day morbidity rates. Although further validations are required particularly to establish generalisability outside of hospitalised children undergoing abdominal surgery. This nutritional profile classification could be used to screen children undergoing surgery and therefore identify patients who (eg, underweight short) might specifically benefit from preoperative nutritional rehabilitation.

\section{Author affiliations}

${ }^{1}$ University of California San Francisco, San Francisco, California, USA

${ }^{2}$ Institute for Digital Research and Education, University of California Los Angeles, Los Angeles, California, USA

${ }^{3}$ British Columbia Children's Hospital, University of British Columbia, Vancouver, British Columbia, Canada

${ }^{4}$ Boston Children's Hospital, Harvard Medical School, Boston, Massachusetts, USA ${ }^{5}$ Nationwide Children's Hospital, The Ohio State University, Columbus, Ohio, USA

${ }^{6}$ Division of Research and Optimal Patient Care, American College of Surgeons, Chicago, Illinois, USA

${ }^{7}$ Department of Surgery, Olin Business School, Center for Health Policy, Washington University School of Medicine in Saint Louis, St. Louis, Missouri, USA

${ }^{8}$ St Louis VA Medical Center, BJC Healthcare Saint Louis, St. Louis, Missouri, USA

${ }^{9}$ Maine Medical Center, Portland, Maine

Contributors Study conception and design were performed by AS, JR-0, EDS and SI. Acquisition of data was performed by BLH, SJR and RLM. Analysis and interpretation of data were performed by AS, JR-0, EDS, SI, SJR, RLM, BLH and AD. Drafting of manuscript was performed by AS, JR-0, EDS, SI, SJR, RLM, BLH and AD. Critical revision was performed by AS, JR-0, EDS, SI, SJR, RLM, BLH and AD.

Funding The authors have not declared a specific grant for this research from any funding agency in the public, commercial or not-for-profit sectors.

Competing interests None declared.

Patient consent Not required.

Ethics approval The RAND Corporation institutional review board approved this study.

Provenance and peer review Not commissioned; externally peer reviewed. 
Data sharing statement These data are from the American College of Surgeons National Surgical Quality Improvement Program Pediatric Internal Dataset. It is similar; however, more extensive than data that is available for all participating NSQIP-P hospitals in North America. Furthermore, the data are proprietary, so very limited people, those who work inside the ACS-NSQIP-P have access to it in the current form used in this manuscript. The use of the algorithms was developed by the researchers in this manuscript and is not available in any of the NSQIP-P datasets.

Open access This is an open access article distributed in accordance with the Creative Commons Attribution Non Commercial (CC BY-NC 4.0) license, which permits others to distribute, remix, adapt, build upon this work non-commercially, and license their derivative works on different terms, provided the original work is properly cited, appropriate credit is given, any changes made indicated, and the use is non-commercial. See: http://creativecommons.org/licenses/by-nc/4.0/.

\section{REFERENCES}

1. Pawellek I, Dokoupil K, Koletzko B. Prevalence of malnutrition in paediatric hospital patients. Clin Nutr 2008;27:72-6.

2. Joosten KF, Hulst JM. Nutritional screening tools for hospitalized children: methodological considerations. Clin Nutr 2014;33:1-5.

3. Karp RJ. The use of the 'at-risk' concept to identify malnourished hospitalized patients: how a two-step process improves clinical acumen. Nutr Clin Pract 1988;3:150-3.

4. Martorell R. Notes on the history of nutritional anthropometry. Fed Proc 1981;40:2572-6.

5. Kvissberg MA, Dalvi PS, Kerac M, et al. Carbohydrate malabsorption in acutely malnourished children and infants: a systematic review. Nutr Rev 2016;74:48-58.

6. Waterlow JC. Protein-energy malnutrition: the nature and extent of the problem. Clin Nutr 1997;16(Suppl 1):3-9.

7. Raval MV, Dillon PW, Bruny JL, et al. Pediatric American College of Surgeons National Surgical Quality Improvement Program: feasibility of a novel, prospective assessment of surgical outcomes. J Pediatr Surg 2011;46:115-21

8. Sharp NE, Knott EM, lqbal CW, et al. Accuracy of American College of Surgeons National Surgical Quality Improvement Program Pediatric for laparoscopic appendectomy at a single institution. $J$ Surg Res 2013;184:318-21.

9. (Internet) WHO, 2017. Global database on child growth and malnutrition. http://www.who.int/nutgrowthdb/about/introduction/en/ index4.html
10. (Internet) CfDCaP, 2017. National center for health statistics. https:// www.cdc.gov/growthcharts/zscore.htm

11. Cohen ME, Ko CY, Bilimoria KY, et al. Optimizing ACS NSQIP modeling for evaluation of surgical quality and risk: patient risk adjustment, procedure mix adjustment, shrinkage adjustment, and surgical focus. J Am Coll Surg 2013;217:336-46.

12. Osborne $\mathrm{NH}$, Ko CY, Upchurch GR, et al. Evaluating parsimonious risk-adjustment models for comparing hospital outcomes with vascular surgery. J Vasc Surg 2010;52:400-5.

13. Merkow RP, Bilimoria KY, Cohen ME, et al. Variability in reoperation rates at 182 hospitals: a potential target for quality improvement. $J$ Am Coll Surg 2009;209:557-64.

14. Mehta NM, Bechard LJ, Cahill N, et al. Nutritional practices and their relationship to clinical outcomes in critically ill childrenan international multicenter cohort study*. Crit Care Med 2012;40:2204-11.

15. de Souza Menezes F, Leite HP, Koch Nogueira PC. Malnutrition as an independent predictor of clinical outcome in critically ill children. Nutrition 2012;28:267-70.

16. Radman M, Mack R, Barnoya J, et al. The effect of preoperative nutritional status on postoperative outcomes in children undergoing surgery for congenital heart defects in San Francisco (UCSF) and Guatemala City (UNICAR). J Thorac Cardiovasc Surg 2014; $147: 442-50$.

17. Mitting R, Marino L, Macrae D, et al. Nutritional status and clinical outcome in postterm neonates undergoing surgery for congenital heart disease. Pediatr Crit Care Med 2015;16:448-52.

18. Keehn A, O'Brien C, Mazurak V, et al. Epidemiology of interruptions to nutrition support in critically ill children in the pediatric intensive care unit. JPEN J Parenter Enteral Nutr 2015;39:211-7.

19. Larsen BM, Goonewardene LA, Field CJ, et al. Low energy intakes are associated with adverse outcomes in infants after open heart surgery. JPEN J Parenter Enteral Nutr 2013;37:254-60.

20. Stey AM, Moss RL, Kraemer K, et al. The importance of extreme weight percentile in postoperative morbidity in children. J Am Coll Surg 2014;218:988-96.

21. Witt CE, Goldin AB, Vavilala MS, et al. Effect of body mass index percentile on pediatric gastrointestinal surgery outcomes. J Pediatr Surg 2016;51:1473-9.

22. Kurtz MP, McNamara ER, Schaeffer AJ, et al. Association of BMI and pediatric urologic postoperative events: Results from pediatric NSQIP. J Pediatr Urol 2015;11:224.e1-6.

23. Shah RK, Stey AM, Jatana KR, et al. Identification of opportunities for quality improvement and outcome measurement in pediatric otolaryngology. JAMA Otolaryngol Head Neck Surg 2014;140:1019-26. 